\title{
ON UNIQUENESS GENERALIZED PROBLEM OF TRICOMI FOR THE CHAPLYGIN EQUATION
}

\author{
Andrey Akimov ${ }^{1 \S}$, Eldar Safin ${ }^{2}$, Alena Agafonova ${ }^{3}$ \\ ${ }^{1,2,3}$ Bashkir State University \\ Sterlitamak Branch \\ 453103, Lenina street 47A, Sterlitamak, RUSSIA
}

\begin{abstract}
In this paper we consider the generalized Tricomi problem in a mixed domain for the Chaplygin equation. Frankl was first which showed that the problem of the expiry of a supersonic jet from a vessel with plane walls in the hodograph plane is reduced to the Tricomi problem for Chaplygin equation. By method of auxiliary functions we received a new theorem of uniqueness of the solution of this problem without any restrictions, except the smoothness on the elliptical part of the border domain.
\end{abstract}

AMS Subject Classification: 35M12

Key Words: Chaplygin equation, Tricomi problem, equation of mixed type

\section{Introduction}

Consider the equation

$$
L z=K(y) z_{x x}+z_{y y}=0,
$$

in an open domain $D$, where $y K(y)>0$ for $y \neq 0$. The domain $D$ is bounded by curves: a piecewise smooth curve $\Gamma$ in the half-plane $y>0$, which intersects the line $y=0$ at the points $A(0,0)$ and $B(l, 0), l>0$; in $y<0, D$ is bounded by two curves: monotonic curve $\gamma_{1}: y=\alpha(x),\left(\alpha^{\prime}(x)<0\right)$ issuing from $A$ and meeting at the point $C$ with charasteristic $\gamma_{2}: \eta=x-\int_{0}^{y} \sqrt{-K(t)} d t=l$, issuing from $B$.

Received: June 20, 2017

Revised: July 5, 2017

Published: August 10, 2017

${ }^{\S}$ Correspondence author (c) 2017 Academic Publications, Ltd. url: www.acadpubl.eu 
Let $K(y) \in C\left[y_{c}, 0\right] \cap C^{2}\left[y_{c}, 0\right), \quad y_{c}$ - the ordinate of point $C$ and let $D_{+}$ be subdomain of $D$ with $y>0$ and $D_{-}$be subdomain of $D$ with $y<0$.

In this paper using a variation of the energy-integral method (abc method) we obtain sufficient conditions for the uniqueness of solution of generalized problem of Tricomi for the Chaplygin equation. It arises in the study of transonic flow, and the proof of uniqueness in this case leads to a proof that continuous transonic flows past smooth profilies do not exist in general [1].

The generalized problem of Tricomi. Find function $z(x, y)$ satisfying the following conditions:

$$
\begin{aligned}
L z(x, y) & \equiv 0,(x, y) \in D_{-} \cup D_{+} ; \\
z(x, y) & \in C(\bar{D}) \cap C^{1}(D) \cap C^{2}\left(D_{-} \cup D_{+}\right) ; \\
\left.z\right|_{\Gamma} & =\varphi(s), \quad 0 \leq s \leq L ; \\
\left.z\right|_{\gamma_{1}} & =\psi(x), \quad 0 \leq x \leq x_{C},
\end{aligned}
$$

where $\varphi$ and $\psi$ are given functions, $L$ - length of curve $\Gamma$.

Definition 1. We call a function regular solution of (1) if the following hold

i) $z(x, y) \in C(\bar{D}) \cap C^{1}(D) \cap C^{2}\left(D_{-} \cup D_{+}\right)$;

ii) we can to applicate Greens theorem to the integrals

$$
\iint_{D} z L z d x d y \iint_{D} z L z_{x} d x d y \iint_{D} z_{y} L z d x d y ;
$$

iii) the boundary integrals which arise exist in the sense that: the limits taken over corresponding interior curves exist as these interior curves approach the boundary.

The question uniqueness of solution of generalized problem of Tricomi for equation of mixed type has been dealt with in the literature by many authors. For an extensive bibliography we refer the reader to [1], [2], [3].

\section{Theorem of Uniqueness}

We introduce Francl's function

$$
F(y)=2\left(\frac{K}{K^{\prime}}\right)^{\prime}+1 .
$$

The following statement is a more general result than Theorem 6, given in [2]. 
Theorem 2. If 1) $K(y) \in C^{2}\left[y_{c}, 0\right), K(0)=0, K^{\prime}(y) \neq 0$ for $y<$ $0, F(0)>0 ; 2)$ there is constant $d>0$ such that $F(y)>-d$ in $\left.D_{-} ; 3\right) z(x, y)$ regular solution of (1) in $D$, 4) $\left.z\right|_{\Gamma \cup \gamma_{1}}=0$, then $z(x, y) \equiv 0$ in $D$.

Proof. Consider the area integral I over domain $D$

$$
I=\iint_{D}\left(a z+b z_{x}+c z_{y}\right)\left(K(y) z_{x x}+z_{y y}\right) d x d y
$$

where $a(x, y), b(x, y), c(x, y)$ are given functions. By (1), the integral I vanishes. We shall show that over $D$ integral I can be made non-positive by proper choice of functions $a(x, y), b(x, y)$ and $c(x, y)$.

Applying Green's formula to the integral (2), similar to the work [3], we get:

$$
\begin{aligned}
0= & \iint_{D}\left[\frac{1}{2}\left(K a_{x x}+a_{y y}\right) z^{2}-a\left(K z_{x}^{2}+z_{y}^{2}\right)-\frac{1}{2} b_{x}\left(K z_{x}^{2}-z_{y}^{2}\right)\right. \\
& \left.-b_{y} z_{x} z_{y}+\frac{1}{2}(K c)_{y} z_{x}^{2}-c_{x} K z_{x} z_{y}-\frac{1}{2} c_{y} z_{y}^{2}\right] d x d y \\
& +\int_{\Gamma+\gamma_{1}+\gamma_{2}}\left[-a z z_{y}+\frac{1}{2} a_{y} z^{2}-b z_{x} z_{y}+\frac{1}{2} c\left(K z_{x}^{2}-z_{y}^{2}\right)\right] d x \\
& +\left[a K z z_{x}-\frac{1}{2} a_{x} K z^{2}+c K z_{x} z_{y}+\frac{1}{2} b\left(K z_{x}^{2}-z_{y}^{2}\right)\right] d y=J_{1}+J_{2} .
\end{aligned}
$$

Choose $b=c \equiv 0$ in $D_{+}$. From $z(x, y)=0$ on $\Gamma \cup \gamma_{1}$ and the fact that $d x=\sqrt{-K} d y\left(\right.$ on $\left.\gamma_{2}\right)$ and $z_{x} d x+z_{y} d y=0 \quad \gamma_{1}$ we get

$$
\begin{aligned}
J_{2}= & \frac{1}{2} \int_{\gamma_{1}}\left(b \frac{d y}{d x}-c\right)\left(\left(\frac{d x}{d y}\right)^{2}+K\right) z_{x}^{2} d x \\
& -\frac{1}{2} \int_{\gamma_{2}}(b+c \sqrt{-K})\left(\sqrt{-K} z_{x}^{2}+2 z_{x} z_{y}+\frac{1}{\sqrt{-K}} z_{y}^{2}\right) d x \\
& -\int_{\gamma_{2}} a \sqrt{-K} z d z-\frac{1}{2} \sqrt{-K} z^{2}\left(a_{x} d x+a_{y} d y\right)=I_{1}+I_{2}+I_{3}, \\
J_{1}= & -\iint_{D_{+}} a\left(K z_{x}^{2}+z_{y}^{2}\right) d x d y+\iint_{D}\left(K a_{x x}+a_{y y}\right) z^{2} d x d y
\end{aligned}
$$




$$
\begin{aligned}
& \quad-\frac{1}{2} \iint_{D_{-}}\left[\left(2 a K+K b_{x}-(K c)_{y}\right) z_{x}^{2}+2 z_{x} z_{y}\left(b_{y}+K c_{x}\right)\right. \\
& \left.+\left(2 a-b_{x}+c_{y}\right) z_{y}^{2}\right] d x d y \\
& =I_{4}+I_{5}+I_{6} .
\end{aligned}
$$

We must choose functions $a(x, y), b(x, y)$ and $c(x, y)$ so that all the integrals $I_{1}, I_{2}, \ldots, I_{6}$ or at least their partial combinations were non-positive. If this occurs then $z(x, y) \equiv 0$ follows immediately.

Follow [6] for $y<0$ choose

$$
c=\frac{4 a K(y)}{K^{\prime}(y)}, \quad b=-c \sqrt{-K(y)} .
$$

Then employing identities (3) we get

$$
I_{1}=-\int_{\gamma_{1}} \frac{2 a K}{K^{\prime}}\left(\frac{d y}{d x}-\sqrt{-K}\right)\left(\frac{d x}{d y}+\sqrt{-K}\right)^{2} z_{x}^{2} d y
$$

Obviously $I_{2}=0$. An integration by parts $I_{3}$ we get

$$
I_{3}=\int_{\gamma_{2}}\left(\sqrt{-K} a_{x}+a_{y}+\frac{a K^{\prime}}{4 K}\right) z^{2} d x
$$

The integral $I_{6}$ is non-positive if the following two conditions hold in $D_{-}$:

$$
\begin{gathered}
\left(K c_{x}+b_{y}\right)^{2} \leq\left(2 a-b_{x}+c_{y}\right)\left(2 a K+K b_{x}-(K c)_{y}\right) \\
2 a+b_{x}-c_{y} \geq 0
\end{gathered}
$$

Obviously, condition (4) holds for all $a(x, y)$. If, now we substitute functions $a(x, y), b(x, y)$ and $c(x, y)$ into (5) we obtain

$$
\sqrt{-K} a_{x}+a_{y}+a \frac{K^{\prime}}{2 K} F(y) \leq 0 .
$$

Similarly as to the work [2] let

$$
a=\left\{\begin{array}{l}
e^{-\beta x}, \quad y \leq 0 \\
e^{-\beta x} \cos \gamma y, \quad y \geq 0
\end{array}\right.
$$

where $\gamma, \beta$ are positive constants .

Obviously $I_{4}$ is non-positive. 
Substituting function $a(x, y)$ into $I_{1}, I_{3}$ and (6) we get

$$
\begin{aligned}
I_{1}+I_{3}= & e^{-\beta x} \int_{\gamma_{2}}\left(-\sqrt{-K} \beta+\frac{K^{\prime}}{4 K}\right) z^{2} d x \\
& e^{-\beta x}-\int_{\gamma_{1}} \frac{2 K}{K^{\prime}}\left(\frac{d y}{d x}-\sqrt{-K}\right)\left(\frac{d x}{d y}+\sqrt{-K}\right)^{2} z_{x}^{2} d y \\
& -\sqrt{-K} \beta+\frac{K^{\prime}}{4 K} F(y) \leq 0 .
\end{aligned}
$$

We choose $\gamma=\frac{\pi}{2 y_{m}}$ (where $y_{m}$ is maximum ordinate in the elliptical domain) and $\beta$ so large that sum of integrals $I_{1}+I_{3}$ is non positive and and hold inequality (7). If $y_{m}$ sufficiently small number then the inequality $I_{5} \leq 0$ holds.

Thus, we can conclude that since the sum of integrals $I_{1}+I_{3}, I_{2}, I_{4}$, $I_{5}, \ldots, I_{6}$ is nonnegative, and each integral is nonpositive, then all the integrals are zero and, in particular, the integral $I_{4}=0$, whence we get that $z(x, y)=0$ in $D^{+}$,and, in particular, $z(x, 0)=0$ and $\frac{\partial z(x, 0)}{\partial y}=0$. Then the uniqueness of the solution of the Cauchy problem implies $z(x, y) \equiv 0$ in $D_{-}$. As a result, we obtain $z(x, y) \equiv 0$ in the domain $D$.

\section{References}

[1] F. Frankl, On the problem of Chaplygin for mixed sub and supersonic flows, Bull. Acad. Sci. USSR, 8 (1945), 195-224.

[2] M.H. Protter, Uniqueness theorems for the Tricomi problem, Indiana Univ. Math. J., 2, No. 1 (1953), doi: 10.1512/iumj.1953.2.52005.

[3] M.M. Smirnov, Equations of mixed type, American Mathematical Society, United States, 1978.

[4] K.B. Sabitov, A.K. Isyangil'din, A Tricomi problem with a nonlocal conjugation condition for the generalized Tricomi equation, Differential Equations, 32, No. 3 (1996), 413.

[5] A. Akimov, G. Galiaskarova, The solution of the Darboux problem for the telegrath equation with deviation from the characteristic, International Journal of Pure and Applied Mathematics, 103, No. 2 (2015), 377-383, doi: 10.12732/ijpam.v103i2.20.

[6] A.A. Akimov, On uniqueness Morawetz problem for the Chaplygin equation, International Journal of Pure and Applied Mathematics, 97, No. 3 (2014), 369-375, doi: 10.12732/ijpam.v97i3.9.

[7] A. Andrey, A. Alena, Analytical solutions for linear Volterra and Abel integral equations of second kind using a power series method, International Journal of Pure and Applied Mathematics, 105, No. 3 (2015), 529-535, doi: 10.12732/ijpam.v105i3.18. 
